\title{
Analysis and research on power distribution law of power generation based on HHT transform
}

\author{
Yuanfeng Huang ${ }^{2}$, Yuze Wang ${ }^{2}$, Xian Cao ${ }^{1,2}$, and Haifeng Wang, ${ }^{2, *}$ \\ ${ }^{1}$ University of Chinese Academy of Sciences, Beijing, China \\ ${ }^{2}$ Institute of Electrical Engineering of the Chinese Academy of Science, Beijing, China
}

\begin{abstract}
In recent years, tidal current power generation technology has achieved rapid development. On the basis of summarizing the characteristics of tidal current power generation, this paper comprehensively analyzes the distribution law of electric energy in tidal current power generation. Hilbert huang transform was applied to the trend of energy in power quality detection and analysis, the key can power on the trend of the measured data grouping processing, and carried out in accordance with the time period, such as day, month and year of HHT analysis, can get tide power distribution, can verify the HHT analysis in the trend of the power law research in the application feasibility and accuracy.
\end{abstract}

\section{Introduction}

With the development of social economy, the demand for energy is increasing day by day. The extensive use of coal, oil and natural gas has also brought about many problems, such as greenhouse effect, environmental pollution and ecological destruction, which will seriously threaten the future survival of human beings [1-2].

Tidal power generation as a new energy generation, whether in grid connection or directly through the power conversion to the load power supply, clear power distribution law of its power generation, in order to better guide its development and utilization. For the research and analysis of the law of electric energy, commonly used methods mainly include: Fourier transform, wavelet transform, S transform, etc., which have their own advantages and disadvantages. Fourier transform is generally used to analyze stable signals, and its extended discrete Fourier transform and fast Fourier transform will produce phase detection error [34].The window function of $S$ transformation cannot be the root function, and it can be adjusted according to the actual needs of specific projects, which is lack of generality and requires a lot of calculation [5-6].Although wavelet transform is applicable to the analysis of non-stationary signals, the selection of wavelet basis is relatively complicated [7].

Hilbert-Huang Transform (HHT) is a nonlinear non-stationary signal processing method which has been developed in recent years. HHT is an adaptive signal analysis method, and its basis function does not need to be set in advance, or its basis function is defined after the fact. It has good flexibility in data processing and overcomes the disadvantage of fixed basis function of wavelet transform [8].

\footnotetext{
* Corresponding author: huangyuanfeng08@mail.iee.ac.cn
} 
In this paper, through analyzing the measured data of tidal current power generation, the power distribution law of tidal current power generation is explored by Hilbert yellow transformation. First of all, the change trend of power generation throughout the day is analyzed in detail. Can then, trend of monthly and annual power generation to HHT analysis of the measured data, get the trend to monthly and annual Hilbert spectrum of electric power and the power transformation trend, through analyzing the Hilbert spectrum can get tide monthly and annual distribution of electric power, and with the test data of monthly and annual electricity distribution curve, verify the feasibility and accuracy of applying HHT analysis in the study of power generation law.

\section{Fundamentals of power generation by power flow}

Tidal energy refers to the kinetic energy of sea water flowing, which is derived from the gravitational attraction between stars and has certain laws to follow. During the new and full moons, the earth, moon and sun line up. At this time, due to the superposition of the gravity of the sun and the moon, the tides generated are relatively high. Such tides are called spring tides, as shown in Fig. 1(a). The local moon and sun form a straight Angle, which is offset by gravity, resulting in low tides, called neap tides, as shown in Fig. 1(b).

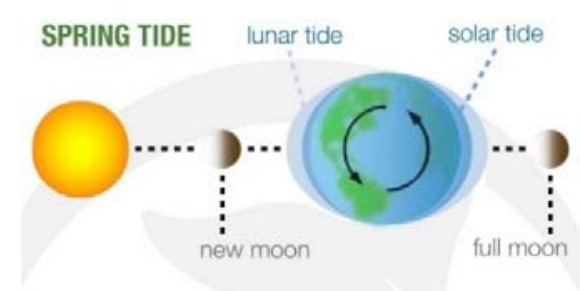

(a) spring tide

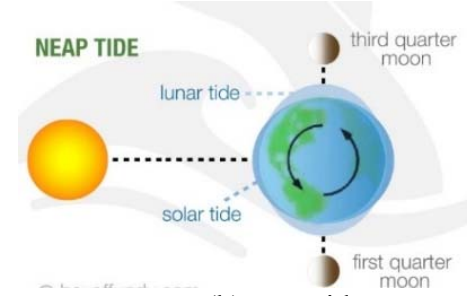

(b) neap tide

Fig. 1. Relative position of earth, moon and day at spring tide and neap tide.

In July 2016, the research team built the "Zhongke Haidian No.1" tidal power generation platform in Zhoushan, Zhejiang Province. Based on this platform, the typical daily power generation data of power flow energy is obtained, as shown in Fig. 2.

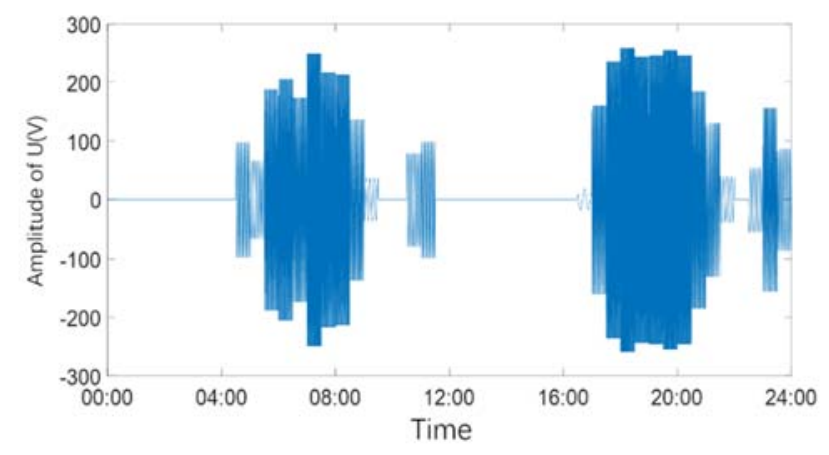

Fig. 2. Daily power output data curve of power flow power generating set.

It can be clearly seen from Fig. 2 that in 24 hours a day, the electric energy distribution experienced four peaks, corresponding to four flows, and experienced the process of rising tide, falling tide, rising tide and falling tide again. The output voltage and power of the motor also have great changes according to different sea water flow rate. 


\section{Hilbert-Huang Transform}

HHT mainly consists of two parts. The first part is Empirical Mode Decomposition (EMD), which was proposed by Huang. The second part is Hilbert Spectrum Analysis (HSA). EMD decomposition plays a crucial role in HHT transformation, which solves the disadvantage of Hilbert transform. EMD decomposition and Hilbert transform, jointly composed of Hilbert yellow transform, have become the best choice method for processing nonlinear nonstationary signals [9].

\subsection{Empirical Mode Decomposition}

EMD is often referred to as a "filtering" process. This filtering process adaptively decomposes any complex signal into a series of Intrinsic Mode Functions (IMF) according to the signal characteristics. IMF is a component function whose instantaneous frequency can be reasonably defined. Its function is locally symmetric about the zero mean, and the number of zero crossings is the same as the number of poles. At the same time, the IMF must meet the following two conditions:

(1) The number of signal extremum points is equal to or one difference from the number of zero points;

(2) The local mean of the upper envelope defined by the maximum and the lower envelope defined by the minimum of the signal is zero.

The EMD screening process is as follows:

(1) For the input signal $x(t)$, obtain the maximum point $x\left(t_{i}\right)$ and minimum point $x\left(t_{j}\right)$;

(2) The upper and lower envelope of the signal is constructed by cubic spline function interpolation for the maximum and minimum points, and the mean function $X L(t)$ of the upper and lower envelope is calculated.

(3) Investigate whether $x l(t)$ meets the IMF conditions. If so, move to the next step; otherwise, carry out the first two steps of XL until the k step meets the IMF conditions, and obtain the first IMF component, denote as $c_{1}$;

(4) Get the first residue $r_{1}=x-c_{1}$, do the above three steps for $r_{1}$, get $c_{2}$, and so on;

(5) until $r_{n}$ is a monotonic signal or there is only one pole.

Finally, the original signal is expressed as

$$
x=\sum_{i=1}^{n} c_{i}+r_{n}
$$

\subsection{Hilbert's analysis}

If $x(t)$ is any signal, the Hilbert transform of $x(t)$ is

$$
y(t)=\frac{1}{\pi} \int_{-\infty}^{+\infty} \frac{x(\tau)}{t-\tau} d \tau
$$

Its inverse Hilbert transform is

$$
x(t)=\frac{1}{\pi} \int_{-\infty}^{+\infty} \frac{y(\tau)}{\tau-t} d \tau
$$

Get the parse signal:

$$
z(t)=x(t)+i y(t)=a(t) e^{j \emptyset(t)}
$$

Where: $a(t)$ is the instantaneous amplitude, and $\emptyset(t)$ is the phase. 


$$
\begin{aligned}
a(t) & =\sqrt{x^{2}(t)+y^{2}(t)} \\
\varnothing(t) & =\arctan \frac{y(t)}{x(t)}
\end{aligned}
$$

The instantaneous frequency is

$$
f(t)=\frac{1}{2 \pi} \frac{d \emptyset(t)}{d t}
$$

Equations (5) and (7) are the expressions of instantaneous amplitude and instantaneous frequency, which are obtained by a simple modulation and demodulation process.

Due to the waveform of its energy, the voltage and frequency of power generation are changing all the time. The instantaneous voltage and frequency obtained by HHT transformation can clearly reflect its changing characteristics.

\section{Analysis of measured data}

\subsection{Analysis of daily distribution law of tidal current power generation}

The data of daily output electric energy of the power flow generating set is shown in Fig. 2. The signals in Fig. 2 were decomposed by EMD, and the first three IMF were selected for analysis, as shown in Fig. 3. Hilbert spectrum of daily power generation data of tidal current energy is shown in Fig. 4.

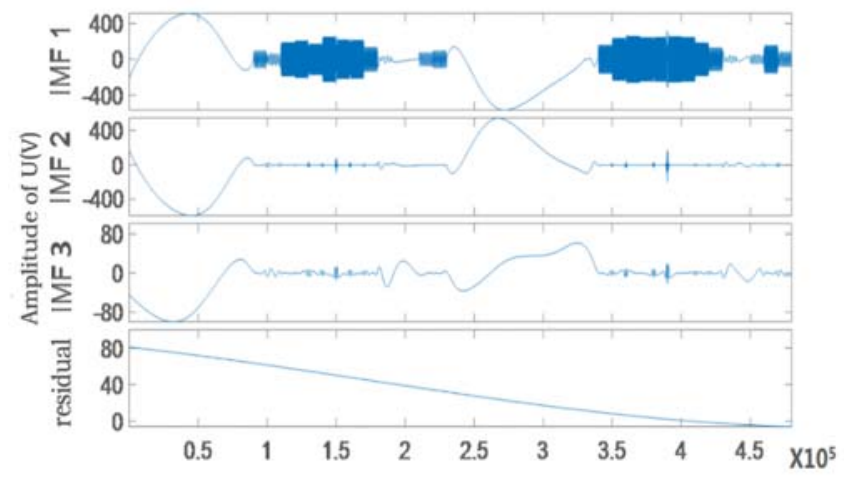

Fig. 3. EMD decomposition of daily power generation data of tidal current energy.

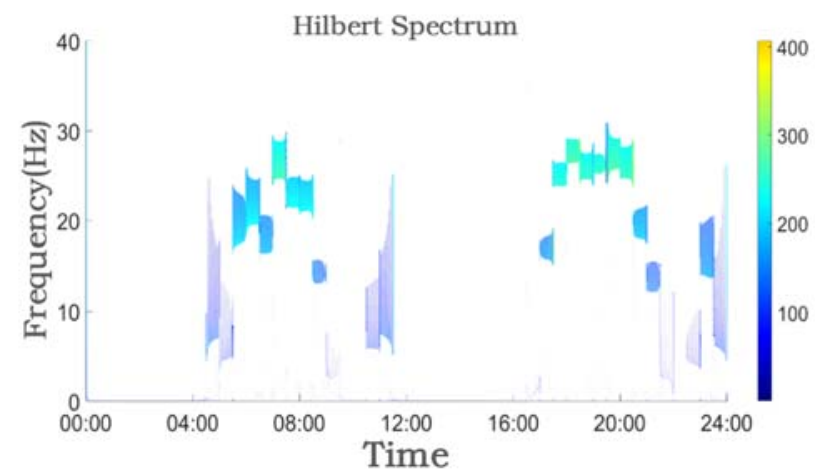

Fig. 4. Hilbert spectrum of daily power generation data of tidal current energy. 
It can be seen from Fig. 3 that the main modes of the signal are concentrated in IMF1, which is caused by the symmetry of the signal. It can also be seen from Fig. 4 that the motor operates in a day, stops running in a day, the maximum voltage appears in a day, and the output voltage exceeds $100 \mathrm{~V}$ in a day, all of which are consistent with Fig. 2.

\subsection{Analysis of monthly power distribution law of power generation}

Based on a $5 \mathrm{~kW}$ tidal current power generation system deployed in the waters of Zhoushan, Zhejiang, using a power analyzer (PW6001 of HIOKI, Japan), the voltage, current, frequency, power and other power quality parameters of the power generation system are detected in real time. The measured data of monthly variation of power generation by tidal current energy is shown in Fig. 5. The EMD decomposition waveform of monthly power generation data of power flow energy is shown in Fig. 6.

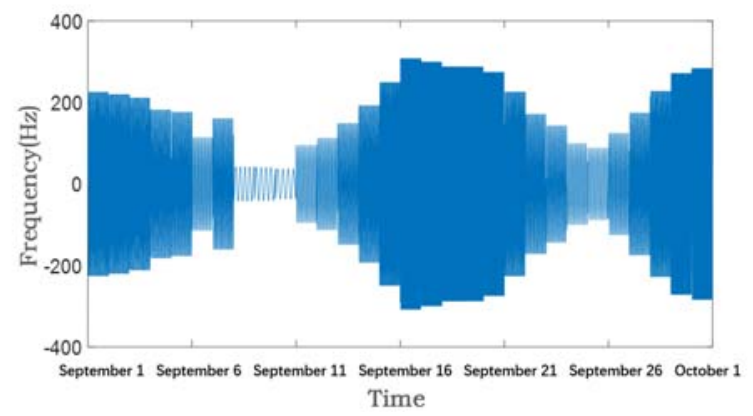

Fig. 5. Monthly power data curve of power flow power generation.

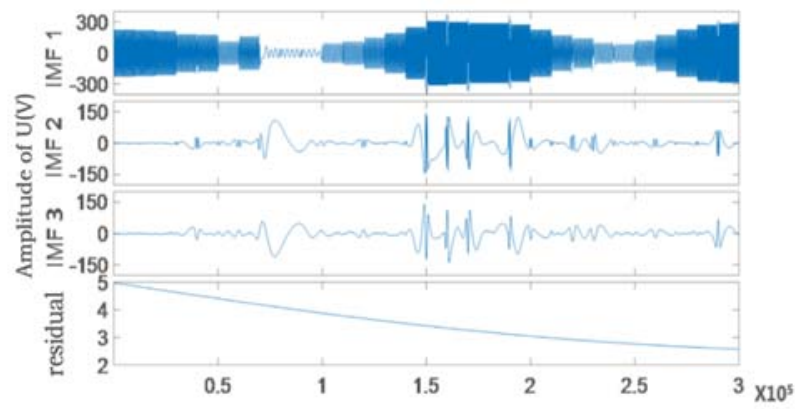

Fig. 6. EMD decomposition of monthly power generation data of power flow.

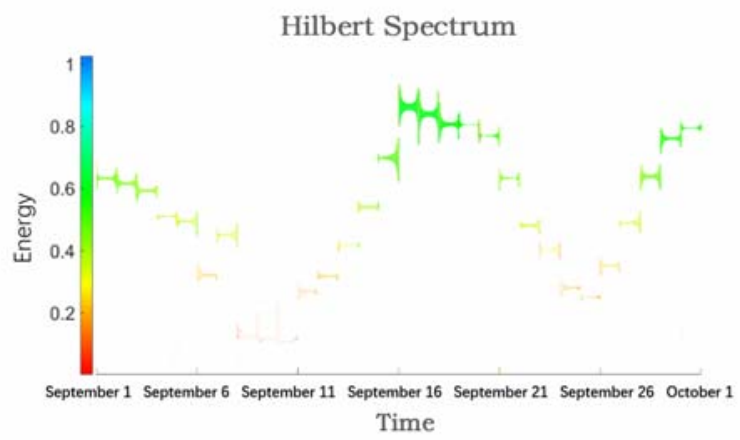

Fig. 7. Hilbert spectrum of monthly power generation data of power flow. 
Hilbert spectrum of monthly power generation data of tidal current energy is shown in Fig. 7. In Figure 7, the electricity generated by the power generation system on the ordinate on different days in a month.

\subsection{Analysis of annual power distribution law of tidal current power generation}

The measured annual voltage distribution data of tidal current power generation are shown in Fig. 8.

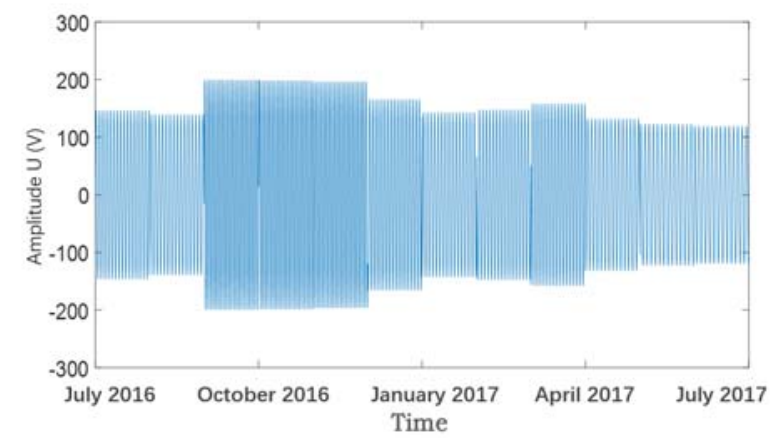

Fig. 8. Annual power data curve of power flow power generation.

EMD decomposition and Hilbert transformation were performed on the data in Fig. 8 respectively, and the obtained results are shown in Fig. 9 and Fig. 10 respectively.

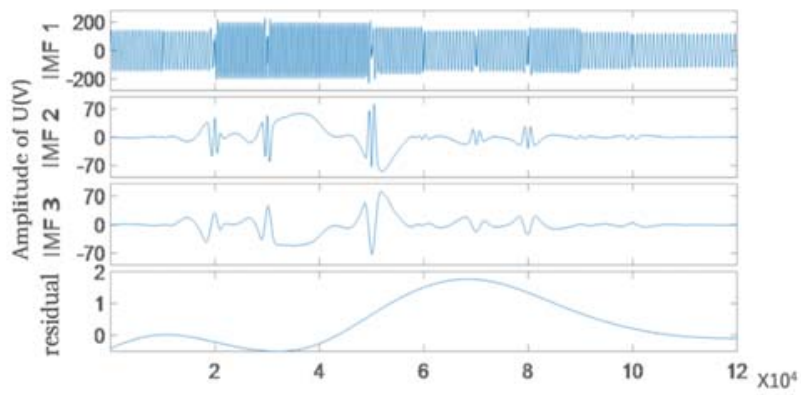

Fig. 9 EMD decomposition of annual power generation data of tidal current energy.

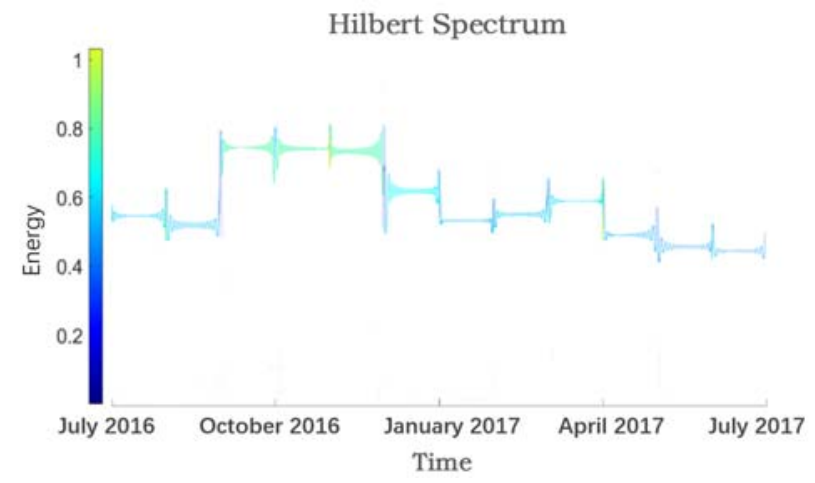

Fig. 10. Hilbert spectrum of annual power generation data of tidal power.

According to the analysis results in Fig. 10, tidal current power generation has the maximum energy in September, October and November of 2016 and the maximum 
corresponding power generation, while it has the lowest energy and the minimum corresponding power generation in April, May and June of 2017.

\section{Conclusion}

Based on Hilbert huang transform can power on the trend analysis of the measured data are processed and analyzed to generating electricity distribution throughout the tide, the tide a day can power the Hilbert spectrum, the trend can power generation from halt to start, from running voltage distribution regularity of the halt process, and it is concluded that the trend can power distribution throughout the day. The long-term power generation distribution law of the tidal current energy system is further analyzed, and the monthly power generation distribution law and the annual power generation energy variation law are obtained.The research results of this paper verify the feasibility and accuracy of applying HHT analysis in the study of power generation law.

\section{Acknowledgment}

This work was supported by National Key Research and Development Project (2018YFB1501905-4) and the Institute of Electrical Engineering, CAS (E1551502)

\section{References}

1. Zhou L, Han Z, Ren J, et al. Current situation and change characteristics of proven oil and gas reserves in China [J]. China Mining Industry, 2019, 28(09):6-11.

2. Gonzalo E. Alvarez. Optimization of the integration among traditional fossil fuels, clean energies, renewable sources, and energy storages: an MILP model for the coupled electric power, hydraulic, and natural gas systems [J]. Computers \& Industrial Engineering.2019.

3. Wen $\mathrm{H}$, Teng $\mathrm{Z}$, Wang $\mathrm{Y}$, et al. Improved windowed interpolation FFT dynamic harmonic analysis algorithm and its application [D]. 2012.

4. $\mathrm{Xu} \mathrm{Y,} \mathrm{Zhao} \mathrm{Y.} \mathrm{Power} \mathrm{quality} \mathrm{disturbance} \mathrm{identification} \mathrm{based} \mathrm{on} \mathrm{short-time} \mathrm{Fourier}$ transform and time location of disturbance based on singular value decomposition [J].Power Grid Technology, 2011, 35(8): 174-180.

5. Yang H, Liu S, Xiao X, Deng W, Chen D. Expert System of Voltage Sag Classification Based on S-transform [J]. Proceedings of the CSEE. 2007(01)

6. Li-Ping Q, Chang-Long H, Jie Z. Research Summary of Power Quality Disturbance Detection and Classification Recognition Method Based on Transform Domain[C]//2020 19th International Symposium on Distributed Computing and Applications for Business Engineering and Science (DCABES). IEEE, 2020: 50-53.

7. Yan Z, Hao C, Chen M, Yang T, Zheng L. Power quality disturbance detection and location based on orthogonal wavelet packet transform [J]. Science and Technology Innovation and Application, 2019(15):16-17.

8. Gao J, Zhu S. Power Quality Detection Method of Active Distribution Network Based on Improved HHT [J].Northeast Electric Power Technology, 2019, 40(07):31-34.

9. Wang K, Wang H, Huang $\mathrm{Y}$, et al. Power quality analysis of tidal current energy generation based on HHT transform[C]//2020 23rd International Conference on Electrical Machines and Systems (ICEMS). IEEE, 2020: 997-1001. 